\title{
SELF-SIMILAR PROCESSES IN COLLECTIVE RISK THEORY
}

\author{
ZBIGNIEW MICHNA ${ }^{1}$ \\ University of Lund \\ Department of Mathematical Statistics \\ Solvegatan 18, Box 118, 22100 Lund, Sweden
}

(Received March, 1997; Revised March, 1998)

\begin{abstract}
Collective risk theory is concerned with random fluctuations of the total assets and the risk reserve of an insurance company. In this paper we consider self-similar, continuous processes with stationary increments for the renewal model in risk theory. We construct a risk model which shows a mechanism of long range dependence of claims. We approximate the risk process by a self-similar process with drift. The ruin probability within finite time is estimated for fractional Brownian motion with drift. A similar model is applicable in queueing systems, describing long range dependence in on/off processes and associated fluid models. The obtained results are useful in communication network models, as well as storage and inventory models.
\end{abstract}

Key words: Risk Process, Long Range Dependence, Ruin Probability, Self-Similar Process, Fractional Brownian Motion, Exponential Bound.

AMS subject classifications: $60 \mathrm{G} 15,60 \mathrm{G} 70,68 \mathrm{M} 20$.

\section{Introduction}

Consider a company which only writes ordinary insurance policies such as accident, disability, health and whole life. The policyholders pay premiums regularly, and at certain random times report claims to the company. A policyholder's premium, the gross risk premium, is a positive amount composed of two components. The net risk premium is the component calculated to cover the payments of claims on the average. The security risk premium, or safety loading, is the component which protects the company from large deviations of claims from the average, and also allows an accumulation of capital. When a claim occurs, the company pays the policyholder a positive amount called the positive risk sum.

As a mathematical model for this situation, we shall assume that claims occur at

\footnotetext{
${ }^{1}$ Supported in part by the Swedish Research Council for Engineering Sciences grant 908911 TFR 91-747.
} 
jumps of a point process $(N(t): t \geq 0)$. While most work in collective risk theory assumes that $N(t)$ is a Poisson process, this restrictive assumption plays no role in our analysis. The successive risk sums $\left(Y_{k}: k \in \mathbb{N}\right)$ are assumed to form a sequence which is stationary and strongly dependent, with $E\left[Y_{k}\right]=\mu>0$. Furthermore, we shall assume that the initial risk reserve of the company is $u>0$, and that the policyholders pay a gross risk premium of $c>0$ per unit time. Thus, the risk process has the form:

$$
R(t)=u+c t-\sum_{k=1}^{N(t)} Y_{k} .
$$

We define the ruin time $T$ as the first time the company has a negative risk reserve:

$$
T=\inf \{t>0: R(t)<0\}
$$

if the set is nonempty, and $T=\infty$ otherwise. In order to avoid $T<\infty$, a.s. we assume the net profit condition $\lim _{t \rightarrow \infty} \frac{E R(t)}{t}>0$ holds. The principal problems of collective risk theory have been to calculate the ruin probability $\Psi(u)=P\{T<$ $\infty \mid R(0)=u\}$. Many of the results for these distributions are complicated expressions which have been obtained using analytical methods. For a comprehensive treatment of the theory up to 1955 , the reader should consult Cramer [8]. A more recent account of the theory is available in Chapter 7 of Takács [44], Grandell [17], and Asmussen [3].

By 1940, Hadwiger [19] was already comparing a discrete-time risk process with a diffusion. This can be viewed, though theoretically not comparable with the modern approach, as the first treatment of diffusion approximations in risk theory. A more modern version, based on weak convergence, is due to Iglehart [20], Grandell [17], and Furrer, Michna and Weron [16]. The basic premise is to let the number of claims grow in a unit time interval, and to make the claim sizes smaller in such a way that the risk process converges weakly to a self-similar process. The idea is to approximate the risk process with a self-similar process with drift. While the classical theory of risk processes requires independence of claims, this assumption can be dropped in our approach. A dependence of claims can guarantee that this model may be similar to a risk model with heavy-tailed claims. As an example of a risk process with such dependence, we construct a risk model in which claims appear in good and bad periods (e.g., good weather and bad weather). We assume that claims in bad periods are bigger than claims in good periods (e.g., the expected value of the claims in bad periods is bigger than the expected value of the claims in good periods). Under natural assumptions on the structure of the good and bad periods, we compute that the claims are strongly dependent.

The use of the results obtained in this paper is motivated by communication network models, as well as storage and inventory models. Traffic on the data networks (e.g., Ethernet LANs) has characteristics substantially different from those of traditional voice traffic. An important feature of data traffic lies in its dependence structure; traditional models are based on assumptions of short-range dependence, while recent measurement and analysis of data traffic has produced strong indications of long-range dependence and self-similarity. Several empirical studies present statistical evidence for existence of these non-standard dependence structures: see for example Heath, Resnick and Samorodnitsky [18]; Leland [24]; Leland and Wilson [25]; Leland, Taqqu, Willinger and Wilson [26, 27]; Norros [30-32]; Willinger, Taqqu, 
Leland and Wilson [47]; Crovella and Bestavros [9]; and Cunha, Bestavros and Crovella [10]. In Norros [30-32], the cumulative traffic process (i.e., the amount of traffic arriving between 0 and $t$ ) is modeled with fractional Brownian motion (the self-similarity parameter $H>\frac{1}{2}$ ).

To study the total traffic for all source-destination network pairs, Willinger, et al. [47] first studied the asymptotic behavior of the integral of the covariance function for one such pair. Their methods (Laplace transform and Tauberian theorem) yield the interesting result that the superposition of a large number of suitably scaled, source-destination pairs is approximately a fractional Brownian motion. In Heath et al. [18], one can find an explanation for the observed long range dependence and selfsimilarity in a simple on/off model applied in communication network models, as well as storage and inventory models.

In applications involving source-destination network pairs, one defines the process $S$ as:

$$
S(t)=\sum_{k=1}^{N(t)} Y_{k}-c t
$$

with $N$ and $\left(Y_{k}: k \in \mathbb{N}\right)$ as above. Consider the process $V(t)=\sup _{0} \leq s \leq t S(s)$ as the workload process of a queueing system, with $N$ representing the time-reversed point process of arrivals of customers, and $\left(Y_{k}: k \in \mathbb{N}\right)$ representing service time in reverse order. Then $V(t)$ is the workload process. We assume that $V$ is generated by a stationary, marked point process $M=\left(\left(T_{k}, U_{k}\right): k \in \mathbb{N}\right)$, such that $T_{k}$ is the time of arrival of the customer, and $U_{k}$ is his service time. By a standard procedure we can extend $M$ to a stationary, marked point process $\left(\left(T_{k}, U_{k}\right): k \in Z\right)$, with doubly infinite time.

In this paper, in contrast to the classical, independent, identically distributed assumptions, we are interested in the case where $\left(Y_{k}: k \in \mathbb{N}\right)$ are strongly dependent. In queueing systems, the relevance of such dependence assumptions is currently receiving much attention as we mentioned above. As an example of a mechanism generating such dependence, one can consider the aforementioned alternating environment.

The paper is organized as follow: Section 2 contains some preliminaries on weak convergence of stochastic processes in the Skorokhod topology and on self-similar processes; in Section 3, we define a sequence of risk processes and show that it converges weakly to a self-similar process with drift; Section 4 deals with the convergence of functionals of the risk process, showing that the finite-time passage probabilities converge; in Section 5, we briefly discuss our approximation when the claim arrival process is a renewal process; and in Section 6, we consider fractional Brownian motion as an example in risk theory. We give an approximation to the ruin probability when the initial capital is sufficiently large.

\section{Preliminaries}

In this section, we assemble those concepts and results from weak convergence theory as those apply to collective risk theory. Furthermore, we define a class $\Pi$ of processes.

Denote by $D$ the space of all cadlag (i.e., right-continuous with left-hand limits) functions on $[0, \infty)$ endowed with the Skorokhod topology (see Ethier and Kurtz [14]). $D[0, \infty)$ is a complete and separable metric space. All stochastic processes in 
this paper are assumed to be in $D$.

Definition 1: A sequence $\left(X^{(n)}: n \in \mathbb{N}\right)$ of stochastic processes is said to converge weakly in the Skorokhod topology to a stochastic process $X$, if for every bounded continuous functional $f$ on $D$, it follows that:

$$
\lim _{n \rightarrow \infty} E\left[f\left(X^{(n)}\right)\right]=E[f(X)]
$$

In this case, one writes $X^{(n)} \Rightarrow X$.

One of the most useful results in weak convergence theory is the continuous mapping theorem. Let $h$ be a measurable mapping of $S$ into another metric space $S^{\prime}$ with $\sigma$-field $\varphi^{\prime}$ of Borel sets. Each probability measure $P$ on $(S, \varphi)$ induces on $\left(S^{\prime}, \varphi^{\prime}\right)$ a unique probability measure $P h^{-1}(A)=P\left(h^{-1} A\right)$ for $A \in \mathcal{Y}^{\prime}$. Let $D_{h}$ be the set of discontinuities of $h$. Then we have:

Proposition 1: (Billingsley [7]) If $P_{n}$ and $P$ are probability measures on $(S, \varphi)$ such that $P_{n} \Rightarrow P$ and $P\left(D_{h}\right)=0$, then $P_{n} h^{-1} \Rightarrow P h^{-1}$.

In collective risk theory, we are interested in sums of a random number of random variables. We also need a general theorem of random change of time. Let $I$ denote the identity function.

Proposition 2: Let $\left(B_{n}: n \in \mathbb{N}\right)$ be processes in $D[0, \infty), B$ be a process with continuous sample paths, and suppose that $B_{n} \Rightarrow B$. Let $\left(N_{n}: n \in \mathbb{N}\right)$ be a sequence of processes with nondecreasing sample paths starting from 0 such that $N_{n} \Rightarrow \lambda I, \lambda>0$. For each $n \in \mathbb{N}, B_{n}$ and $N_{n}$ are assumed to be on the same probability space. Then:

$$
B_{n}\left(N_{n}\right) \Rightarrow B(\lambda I)
$$

Proof: The process $B$ has continuous sample paths so the assertion is an immediate application of the method used in Billingsley [7].

The concept of semi-stability was introduced by Lamperti [22]. Mandelbrot and Van Ness [29] call it self-similarity when appearing in conjunction with stationary increments as it does here.

Definition 2: A process $Z_{H}$ possesses properties $\Pi$ (i.e., $Z_{H} \in \Pi$ ), if for some $0<H \leq 1$ :

1. $Z_{H}(0)=0$ a.s.

2. $Z_{H}$ has strictly stationary increments, that is the random function $M_{h}(t)=Z_{H}(t+h)-Z_{H}(t), h \geq 0$, is strictly stationary.

3. $\quad Z_{h}$ is self-similar of order $H(H-s s)$, that is:

$$
Z_{H}(c t) \stackrel{d}{=} c^{H} Z_{H}(t)
$$

in the sense of finite-dimensional distributions.

4. $E Z_{H}(t)=0$ and $E\left|Z_{H}(t)\right|^{\gamma}<\infty$ for $\gamma \leq \frac{1}{H}$.

5. $Z_{H}$ is a.s. continuous.

Examples of such a process are fractional Brownian motion [Gaussian process] and the Rosenblatt process [non-Gaussian process] (see Mandelbrot and Van Ness [29] and Rosenblatt [35]). If not stated otherwise explicitly, we make the following assumptions for the rest of the paper.

Assumption 1: The parameter of self-similarly satisfies:

$$
\frac{1}{2}<H<1
$$




\section{Weak Convergence of Risk Reserve Processes}

We shall now construct a sequence of risk processes and show that it converges to a process $Z_{H}$ with drift where $Z_{H} \in \Pi$.

The sequence $\left(Q^{(n)}: n \in \mathbb{N}\right)$ of risk processes is given as follows: for every $n \in \mathbb{N}$ let $u^{(n)}>0$ denote the initial risk reserve, $c^{(n)}>0$ the premium rate, and $N^{(n)}$ the corresponding point process. The claim sizes are denoted by $\left(Y_{k}^{(n)}: k \in \mathbb{N}\right)$. Then:

$$
Q^{(n)}(t)=u^{(n)}+c^{(n)} t-\sum_{k=1}^{N^{(n)}(t)} Y_{k}^{(n)}
$$

We assume that the claims are of the form $Y_{k}^{(n)}=\frac{1}{\varphi(n)} Y_{k}$, where $\left(Y_{k}: k \in \mathbb{N}\right)$ is a stationary sequence with common distribution function $F$ and mean $\mu$ such that:

$$
\frac{1}{\varphi(n)} \sum_{k=1}^{[n t]}\left(Y_{k}-\mu\right) \Rightarrow Z_{H}(t),
$$

where $\varphi(n)=n^{H} L(n)$, and the function $L$ is slowly varying at infinity. To construct such a sequence (see Taqqu [46]), let us take a stationary Gaussian sequence $\left(X_{k}\right.$ : $k \in \mathbb{N}$ ) with $E\left[X_{i}\right]=0$ and $E\left[X_{i}^{2}\right]=1$. Let $G$ be a real-valued measurable function such that $G\left(X_{i}\right)$ has mean 0 and finite variance. As mentioned above, we shall focus on values of $H$ satisfying $\frac{1}{2}<H<1$. We assume $E\left[X_{i} X_{i+k}\right] \sim k^{-D} L(k)$ as $k \rightarrow \infty$ for some slowly varying function $L$ and some constant $D>0 . \quad H>\frac{1}{2}$ arises when $D<\frac{1}{m}$, where $m$, the Hermite rank of $G$, is the index of the first non-zero coefficient in the Hermit polynomials expansion of $G$. Under these assumptions, $\sum_{k=1}^{\infty} E\left[G\left(X_{i}\right) G\left(X_{i+k}\right)\right]=\infty$, and the sequence $\left(G\left(X_{i}\right): i \in \mathbb{N}\right)$ is so strongly dependent that the limit of

$$
Z^{(n)}(t)=\frac{1}{\varphi(n)} \sum_{k=1}^{[n t]} G\left(X_{k}\right)
$$

may not be Gaussian.

Now we construct an insurance model, which as assumed above, produces strongly dependent claims.

We assume that we have good periods and bad periods when we observe arriving claims (e.g., periods of good weather and periods of bad weather). These two periods alternate. Let $\left(T^{G}, T_{n}^{G}, n \in \mathbb{N}\right)$ be independent, identically distributed non-negative random variables representing good periods; similarly, let $\left(S_{B}, S_{n}^{B}, n \in \mathbb{N}\right)$ be independent, identically distributed non-negative random variables representing bad periods. The $T$ 's are assumed independent of the $S$ 's, the common distribution of good periods is $F^{G}$, and the distribution of bad periods is $F^{B}$. We assume both $F^{G}$ and $F^{B}$ have finite means $\nu_{G}$ and $\nu_{B}$, respectively, and we set $\nu=\nu_{G}+\nu_{B}$.

Consider the pure renewal sequence initiated by a good period $\left(0, \sum_{i=1}^{n}\left(T_{i}^{G}+S_{i}^{B}\right), n \in \mathbb{N}\right)$. The interarrival distribution is $F^{G} * F^{B}$ and the mean interarrival time is $\nu$. This pure renewal process has stationary version (see Asmussen [2]) $\left[D, D+\sum_{i=1}^{n}\left(T_{i}^{G}+S_{i}^{B}\right), n \in \mathbb{N}\right]$, where $D$ is a delay random variable. However, by defining the initial delay interval of length $D$ this way, the interval does not decompose into a good and a bad period the way subsequent interarrival intervals do. 
Consequently, we turn to an alternative construction of the stationary renewal process (see Heath et al. [18]).

Define three independent random variables $B, T_{0}^{G}$ and $S_{0}^{G}$, which are independent of $\left(S^{B}, T_{n}^{G}, S_{n}^{G}, n \in \mathbb{N}\right)$ as follows: $B$ is a Bernoulli random variable with values in $\{0,1\}$ and mass functions

$$
P\{B=1\}=\frac{\nu_{G}}{\nu}=1-P\{B=0\}
$$

and $(x>0)$, where

$$
\begin{aligned}
& P\left\{T_{0}^{G}>x\right\}=\int_{x}^{\infty} \frac{1-F^{G}(s)}{\nu_{G}} d s=: 1-F_{0}^{G}(x), \\
& P\left\{S_{0}^{B}>x\right\}=\int_{x}^{\infty} \frac{1-F^{B}(s)}{\nu_{B}} d s=: 1-F_{0}^{B}(x) .
\end{aligned}
$$

Define a delay random variable $D_{0}$ by

$$
D_{0}=\left(T_{0}^{G}+S^{B}\right) B+(1-B) S_{0}^{B}
$$

and a delayed renewal sequence by

$$
\left(S_{n}, n \geq 0\right):=\left(D_{0}, D_{0}+\sum_{i=1}^{n}\left(T_{i}^{G}+S_{i}^{B}\right), n \geq 0\right) .
$$

One can verify that this delayed renewal sequence is stationary (see Heath et al. [18]).

We now define $L(t)$ to be 1 if $t$ falls in a good period, and $L(t)=0$ if $t$ is in a bad period. More precisely, the process $(L(t), t \geq 0)$ is defined in terms of $\left(S_{n}, n \geq 0\right)$ as follows:

$$
L(t)=B \boldsymbol{I}_{\left[0, T_{0}^{G}\right)}(t)+\sum_{n=0}^{\infty} \boldsymbol{I}_{\left[S_{n} \leq t<S_{n}+T_{n+1}^{G}\right)}
$$

Proposition 3: The process $(L(t), t \geq 0)$ is strictly stationary and

$$
P\{L(t)=1\}=E L(t)=\frac{\nu_{G}}{\nu} .
$$

Proof: See Heath et al. [18], Proposition 2.1.

Let $\left(Y_{n}^{G}, n \geq 0\right)$ be independent, identically distributed random variables representing claims appearing in good periods (e.g., $Y_{n}^{G}$ describes a claim which may appear at the $n$th moment in a good period). Similarly, let $\left(Y_{n}^{B}, n \geq 0\right)$ be independent, identically distributed random variable representing claims appearing in bad periods (e.g., $Y_{n}^{B}$ describes a claim which may appear at the $n$th moment in a bad period). We assume that $\left(Y_{n}^{G}, n \geq 0\right),\left(Y_{n}^{B}, n \geq 0\right)$ and $(L(t), t \geq 0)$ are independent, $E\left[Y_{0}^{G}\right]=g$ and $E\left[Y_{0}^{B}\right]=b(g<b)$, and the second moments of $Y_{0}^{G}$ and $Y_{0}^{B}$ exist. Then the claim $Y_{n}$ appearing at the $n$th moment $(n \geq 0)$ is

$$
Y_{n}=L(n) Y_{n}^{G}+(1-L(n)) Y_{n}^{B}
$$


The sequence $\left(Y_{n}, n \geq 0\right)$ is stationary.

Proposition 4: Assume that

$$
1-F^{G}(t)=t^{-(D+1)} K(t), \quad t \rightarrow \infty
$$

$0<D<1$, where $K$ is slowly varying at infinity. Assume, moreover, that

$$
1-F^{B}(t)=o\left(1-F^{G}(t)\right), \quad t \rightarrow \infty,
$$

and there is an $n \geq 1$ such that $\left(F_{G} * F_{B}\right)^{n *}$ is nonsingular. Then

$$
\operatorname{Cov}\left(Y_{0}, Y_{n}\right) \sim \frac{\nu_{B}^{2}(b-g)^{2}}{D \nu^{3}} n-D_{K}(n)
$$

when $n \rightarrow \infty$.

Proof: Let us notice that

$$
\operatorname{Cov}\left(Y_{0}, Y_{n}\right)=(b-g)^{2} \operatorname{Cov}(L(0), L(n))
$$

and

$$
\operatorname{Cov}(L(0), L(n)) \sim \frac{\nu_{B}^{2}}{D \nu^{3}} n^{-D} K(n),
$$

when $n \rightarrow \infty$ (see Heath et al. [18], Theorem 4.3). From this, (11) follows.

We assumed that the good period dominates the bad period but one can approach the problem reversely, (e.g., the bad period can dominate the good period). One can see the symmetry of this good and bad period characteristics in the covariance function (see Heath et al. [18]). This same argument can be used on/off models and associated fluid models.

Let us determine the limiting process of $Z^{(n)}$ given in $(6)$. It is sufficient to study the convergence of the finite-dimensional distributions of process $Z^{(n)}$, when $G=H_{m}$, where $H_{m}$ denotes the Hermite polynomial of order $m$.

When $m=1, Z^{(n)}$ converges weakly to fractional Brownian motion $B_{H}$ with parameter $\frac{1}{2}<H=1-\frac{D}{2}<1$. This limiting process is Gaussian, with zero mean and $E\left|B_{H}(t)-B_{H}(s)\right|^{2}=|t-s|^{2 H}$. The process is defined for $0<H<1$. It is Brownian motion when $H=\frac{1}{2}$. For a detailed treatment of $B_{H}$, see Mandelbrot and Van Ness [29], and Samorodnitsky and Taqqu [36].

When $m=2, Z^{(n)}$ converges weakly to the non-Gaussian process called the Rosenblatt process (see Rosenblatt [35]).

Partial results for $m=3$, where the limiting process is not Gaussian, are given in Taqqu [45].

We put $Y_{k}=G\left(X_{k}\right)+\mu$ for $k \in \mathbb{N}$.

We define the process $(Q(t): t \geq 0)$ by

$$
Q(t)=u+c t-\lambda^{H} Z_{H}(t)
$$

where $u$ and $c$ are positive numbers, and $\left(Z_{H}(t): t \geq 0\right)$ is a process endowed with properties $\Pi(H)$. Here $\lambda$ is some positive constant which will be specified in the next theorem. The following theorem shows that the sequence $\left(Q^{(n)}: n \in \mathbb{N}\right)$ converges weakly to the process in (12): 
Theorem 3: Let the sequence $\left(Y_{k}: k \in \mathbb{N}\right)$ be as above and let $\left(N^{(n)}: n \in \mathbb{N}\right)$ be a sequence of point processes such that

$$
\frac{N^{(n)}(t)-\lambda n t}{\varphi(n)} \rightarrow 0
$$

in probability in the Skorokhod topology for some positive constant $\lambda$. Assume also that

$$
\lim _{n \rightarrow \infty}\left(c^{(n)}-\lambda n \frac{\mu}{\varphi(n)}\right)=c
$$

and

$$
\lim _{n \rightarrow \infty} u^{(n)}=u
$$

Then

$$
u^{(n)}+c^{(n)} t-\frac{1}{\varphi(n)} \sum_{k=1}^{N^{(n)}(t)} Y_{k} \Rightarrow u+c t-\lambda^{H} Z_{H}(t)
$$

in the Skorokhod topology as $n \rightarrow \infty$.

Proof: Let us write the process $Q^{(n)}(t)$ in the following form:

$$
\begin{gathered}
Q^{(n)}(t)=u^{(n)}+c^{(n)} t-\frac{1}{\varphi(n)} \sum_{k=1}^{N^{(n)}(t)} Y_{k} \\
=u^{(n)}+t\left(c^{(n)}-\lambda n \frac{\mu}{\varphi(n)}\right)-\mu\left(\frac{N^{(n)}(t)-\lambda n t}{\varphi(n)}\right)-\frac{1}{\varphi(n)} \sum_{k=1}^{N^{(n)}(t)}\left(Y_{k}-\mu\right) .
\end{gathered}
$$

From the assumption in (13), we obtain

$$
\mu\left(\frac{N^{(n)}(t)-\lambda n t}{\varphi(n)}\right) \rightarrow 0
$$

in probability in the Skorokhod topology as $n \rightarrow \infty$. From (13) and Proposition 2 we obtain that

$$
\frac{1}{\varphi(n)} \sum_{k=1}^{N^{(n)}(t)}\left(Y_{k}-\mu\right) \Rightarrow \lambda^{H} Z_{H}(t)
$$

in the Skorokhod topology as $n \rightarrow \infty$. Because

$$
u^{(n)}+t\left(c^{(n)}-\lambda n \frac{\mu}{\varphi(n)}\right)-\mu\left(\frac{N^{(n)}(t)-\lambda n t}{\varphi(n)}\right)
$$

converges to $u+c t$ in probability in the Skorokhod topology, the proof is complete.

The distribution of a risk process can be approximated by the distribution of process in (12). 


\section{The Convergence of Functionals of Risk Processes}

Collective risk theory has mainly been concerned with functionals which represent the total assets of the insurance company at time $t$, namely $Q^{(n)}$, and with the ruin time $T^{(n)}$, which is defined as $T^{(n)}=T\left(Q^{(n)}\right)$, where

$$
T(x)=\inf \{t>0: x(t)<0\}
$$

if the set $\{t>0: x(t)<0\}$ is not empty, and $+\infty$ otherwise. We need a theoretical result which permits us to approximate the finite-time ruin probability by the ruin probability of the corresponding process $Q$. The process given in (12) has continuous sample paths. It is known that the convergence to a continuous function in the Skorokhod topology is equivalent to uniform convergence on compacts. Using Proposition 1, we get:

Proposition 5: Let $T$ be the functional defined in (17). If $Q^{(n)} \Rightarrow Q$, with $Q$ being given in (12), and

$$
P\left\{\inf _{0 \leq s \leq t} Q(s)=0\right\}=0
$$

for all $t>0$, then

$$
T\left(Q^{(n)}\right) \Rightarrow T(Q)
$$

Proof: Let $x_{n}$ converge to $x$ in the Skorokhod topology, where $x$ is a continuous trajectory of the process $Q$. Then $x_{n}$ tends to $x$ uniformly on compacts. First assume that $T(x)=\infty$. This means that $x(t)>0$ for all $t>0$ because we assumed that $P\left\{\inf _{0}<s<t Q(s)=0\right\}=0$ for all $t>0$. Let $N$ be such that, for sufficiently large $n, x_{n}(\bar{t})>0$ for all $0 \leq t \leq N$. Letting $N \rightarrow \infty$, we obtain that $T\left(x_{n}\right) \rightarrow \infty$ as $n \rightarrow \infty$.

Now let $T(x)<\infty$, and assume that $T\left(x_{n}\right) \rightarrow T$ and $T>T(x)$ (more precisely there is such a subsequence of $\left.\left\{T\left(x_{n}\right)\right\}\right)$. Then there is $\delta>0$ such that $x(T(x)+$ $\delta)<0$ and $T(x)+\delta<T$. Since $x_{n}(T(x)+\delta)<0$ for sufficiently large $n$, this is a contradiction.

Remark: Proposition 3 shows that the finite-time ruin probabilities converge:

$$
\lim _{n \rightarrow \infty} P\left\{T\left(Q^{(n)}\right) \leq t\right\}=P\{T(Q) \leq t\}
$$

or equivalently,

$$
\lim _{n \rightarrow \infty} P\left\{\inf _{0 \leq s \leq t} Q^{(n)}(s)<0\right\}=P\left\{\inf _{0 \leq s \leq t} Q(s)<0\right\} .
$$

Hence, we can approximate the finite-time ruin probabilities by the probability of the first 0 -downcrossing of the process in (12). However, it is not clear whether the convergence of the infinite-time ruin probabilities holds, i.e., whether:

$$
\inf _{0 \leq s} Q^{(n)}(s) \underset{0 \leq s}{\rightarrow} \inf Q(s)
$$

In Proposition 3, we proved much more (i.e., we proved that if $Q_{n}$ converges almost surely to $Q$ then $T\left(Q_{n}\right) \rightarrow T(Q)$ a.s. $)$. 


\section{A Renewal Type Model}

To construct an example of risk processes which converge to a self-similar process, we have to check the conditions of Theorem 3. We consider the case where the occurrence of the claims is described by a renewal process $N$ :

$$
N(t)=\max \left\{n: \sum_{k=1}^{n} T_{k} \leq t\right\} .
$$

The inter-occurrence times $\left(T_{k}: k \in \mathbb{N}\right)$ are assumed to be independent, positive random variables. We define

$$
N^{(n)}(t)=N(n t)
$$

Let $B=(B(t): t \geq 0)$ denote standard one-dimensional Brownian motion. Then the following proposition holds:

Proposition 6: Let $(N(t): t \geq 0)$ be a renewal process with inter-occurrence times $\left(T_{k}: k \in \mathbb{N}\right)$, and assume that there exists a positive constant $\lambda$ such that

$$
\frac{1}{\psi(n)} \sum_{k=1}^{[n t]}\left(T_{k}-\frac{1}{\lambda}\right) \Rightarrow B(t)
$$

in the Skorokhod topology as $n \rightarrow \infty$, where $\psi(n)=n^{1 / 2} L(n)$ ( $L$ is slowly varying at infinity). Then, for $1 / 2<H<1$, we have:

$$
\frac{N(n t)-\lambda n t}{n^{H}} \rightarrow 0
$$

in probability in the Skorokhod topology.

Proof: It suffices to check that

converges to 0 as $n \rightarrow \infty$. If

$$
P\left\{\sup _{0 \leq s \leq t} \frac{|N(n s)-\lambda n s|}{n^{H}}>\epsilon\right\}
$$

$$
\sup _{0 \leq s \leq t} \frac{|N(n s)-\lambda n s|}{n^{H}}>\epsilon,
$$

then there exists $s$ such that $0 \leq s \leq t$ and

$$
N(n s)-\lambda n s>\epsilon n^{H}
$$

or

$$
N(n s)-\lambda n s<=\epsilon n^{H}
$$

From the inequality in (23) we obtain

$$
\sum_{k=1}^{\left[\lambda n s+\epsilon n^{H}\right]} T_{k}<n s .
$$

Let us define $u$ such that $n u=\lambda n s+\epsilon n^{H}$. Then 


$$
\sum_{k=1}^{[n u]}\left(T_{k}-\frac{1}{\lambda}\right)<-\frac{\epsilon n^{H}}{\lambda}
$$

Therefore,

$$
\sup _{0 \leq u \leq \lambda t+\epsilon n^{H-1}} \frac{1}{n^{H}}\left|\sum_{k=1}^{[n u]}\left(T_{k}-\frac{1}{\lambda}\right)\right|>\frac{\epsilon}{\lambda}
$$

Let us notice that if $0<H<1$, then the above supremum is attained on bounded intervals. Because $H>1 / 2$, the assumption in (21) implies that

$$
\frac{1}{n^{H}} \sum_{k=1}^{[n u]}\left(T_{k}-\frac{1}{\lambda}\right) \underset{n \rightarrow \infty}{\rightarrow} 0
$$

in probability in the Skorokhod topology. In the second case (see (24)), we obtain

For $n u=\lambda n s-\epsilon n^{H}$ we have

$$
\sum_{k=1}^{\left[\lambda n s-\epsilon n^{H}\right]} T_{k}>n s .
$$

$$
\sum_{k=1}^{[n u]}\left(T_{k}-\frac{1}{\lambda}\right)>\frac{\epsilon n^{H}}{\lambda}
$$

Thus,

$$
\sup _{0 \leq u \leq \lambda t-\epsilon n^{H-1}} \frac{1}{n^{H}}\left|\sum_{k=1}^{[n u]}\left(T_{k}-\frac{1}{\lambda}\right)\right|>\frac{\epsilon}{\lambda} .
$$

Finally, from(25) we have

$$
P\left\{\sup _{0 \leq s \leq t} \frac{|N(n s)-\lambda n s|}{n^{H}}>\epsilon\right\} \underset{n \rightarrow \infty}{\rightarrow} 0
$$

and the proof is complete.

Note that $(21)$ is true in the ordinary renewal theory situation where $\left(T_{k}\right)$ is a sequence of independent, identically distributed random variables with mean $\frac{1}{\lambda}$ and variance $\sigma^{2}$. In this case, we have

$$
\frac{N(n t)-\lambda n t}{\sqrt{n}} \Rightarrow \sigma \lambda^{3 / 2} B(t) .
$$

(see Billingsley [7]).

\section{An Example: Fractional Brownian Motion}

First we recall the following definition:

Definition 3: A stochastic process $B_{H}=\left(B_{H}(t): t \geq 0\right)$ is called fractional Brownian motion if

$B_{H}(t)=C_{H}\left[\int_{-\infty}^{0}\left\{(t-y)^{H-1 / 2}-(-y)^{H-1 / 2}\right\} d B(y)+\int_{0}^{t}(t-y)^{H-1 / 2} d B(y)\right]$, 
where $0<H<1, H$ is the parameter of self-similarity, $C_{H}$ is a real constant depending on $H$, and $B$ is a standard Brownian motion defined on the whole real line.

The process $B_{H}$ possesses properties $\Pi$. Observe that $B_{H}$ with $H=\frac{1}{2}$ is Brownian motion, and that in general, the process $B_{H}$ has stationary, but not independent increments. The fractional Brownian motion process $B_{H}$ is a very important generalization of Brownian motion because $B_{H}$ is the only Gaussian $H-s s$ process with stationary increments. The fractional Brownian motion has expectation $E\left[B_{H}(t)\right]=0$ and covariances $E\left[B_{H}(t) B_{H}(s)\right]=\frac{1}{2} \sigma^{2}\left\{|t|^{2 H}+|s|^{2 H}-|t-s|^{2 H}\right\}$, where $E\left[B_{H}^{2}(1)\right]=\sigma^{2}$. For more details, see Mandelbrot and Van Ness [29] and Samorodnitsky and Taqqu [36].

We shall use the standard notation $\Phi(z)$ and $\phi(z)$ for the standard normal distribution and density functions, respectively. We recall the elementary relation:

$$
1-\Phi(x) \sim x^{-1} \phi(x)
$$

for $x \rightarrow \infty$, and

$$
1-\Phi(x+y / x) \sim[1-\Phi(x)] e^{-y}
$$

for $y$ fixed and $x \rightarrow \infty$.

Let us define

$$
Q(t)=u+c t-\lambda^{H} B_{H}(t)
$$

where $u$ and $c$ and $\lambda$ are positive constants. Recall that we assume $\frac{1}{2}<H<1$. Our main aim is to find the ruin probability of the process in (30). We need bounds or limit theorems for the ruin probability of process $B_{H}$ because we do not know the exact form of this probability. This will be made by applying the easy consequence of the Normal Comparison Lemma (Slepian [42], see also Corollary 4.2.3 in Leadbetter et al. [23]) and the continuity of the sample paths.

Lemma 1: Let $X_{1}$ and $X_{2}$ be Gaussian continuous processes. Suppose that for $T>0$ we have $E\left[X_{1}(s) X_{1}(t)\right] \geq E\left[X_{2}(s) X_{2}(t)\right], E\left[X_{1}(t)\right]=0, E\left[X_{2}(t)\right]=0$, and $E\left[X_{1}^{2}(t)\right]=E\left[X_{2}^{2}(t)\right]$ when $0 \leq s, t \leq T$. Then

$$
P\left\{\sup _{0 \leq s \leq T}\left(X_{1}(s)-c s\right)>u\right\} \leq P\left\{\sup _{0 \leq s \leq T}\left(X_{2}(s)-c s\right)>u\right\} .
$$

Proof: This follows from Corollary 4.2.3 of Leadbetter et al. [23] and continuity of sample paths.

We give another lemma:

Lemma 2: Let $B$ be a standard Brownian motion, and $u \geq 0, c \geq 0$. Then

$$
P\left\{\inf _{s \geq 0}(u+c s+B(s))<0\right\}=\exp (-2 u c) .
$$

Proof: See Grandell [17].

Now we state a theorem which enables us to estimate the ruin probability of the process in (30) for an arbitrary amount of initial capital:

Theorem 6: Let $Q$ be the process given in (30). Then

$$
P\left\{T(Q) \leq t \leq 1-\Phi\left(\frac{u+c t}{\sigma(\lambda t)^{H}}\right)+\exp \left(\frac{-2 u c t}{\sigma^{2}(\lambda t)^{2 H}}\right)\left[1-\Phi\left(\frac{u-c t}{\sigma(\lambda t)^{H}}\right)\right],\right.
$$


where the functional $T$ is given in (17), and $\sigma^{2}=E\left[B_{H}^{2}(1)\right]$.

Proof: For simplicity, assume $E\left[B_{H}^{2}(1)\right]=1$ and $\lambda=1$. Let $B$ be standard Brownian motion and define the Gaussian process

$$
Y(s)=B\left(s^{2 H}\right)
$$

for $s \geq 0$. It is clear that

$$
\begin{aligned}
& E[Y(s)]=0, \quad E\left[Y^{2}(s)\right]=s^{2 H} \\
& E[Y(s) Y(t)]=s^{2 H}, \quad 0 \leq s \leq t .
\end{aligned}
$$

From the convexity of function $t^{2 H}\left(\frac{1}{2}<H<1\right)$, it follows that

$$
E\left[B_{H}(s) B_{H}(t)\right] \geq E[Y(s) Y(t)]
$$

Hence, by Lemma 1 it follows from (36) and (35) that

$$
P\left\{\sup _{0 \leq s \leq t}\left(B_{H}(s)-c s\right)>u\right\} \leq P\left\{\sup _{0 \leq s \leq t}(Y(s)-c s)>u\right\} .
$$

So we obtain

$$
\begin{aligned}
& P\left\{\inf _{0 \leq s \leq t}\left(u+c s-B_{H}(s)\right)<0\right\} \leq P\left\{\inf _{0 \leq s \leq t}(u+c s-Y(s))<0\right\} \\
& =P\left\{\inf _{0 \leq s \leq t^{2 H}}\left(u+c s^{\frac{1}{2 H}}+B(s)\right)<0\right\} \\
& =P\left\{\inf _{s \geq t^{-2 H}}\left(u+c s^{-\frac{1}{2 H}}+B(1 / 2)\right)<0\right\} \\
& =P\left\{\inf _{s \geq t}\left(u s+c s^{1=\frac{1}{2 H}}+s B(1 / s)\right)<0\right\} \\
& =P\left\{\inf _{s \geq t}\left(u s+c s^{1-\frac{1}{2 H}}+B(s)\right)<0\right\} .
\end{aligned}
$$

The last equality above follows since $s B(1 / s)$ is also a standard Brownian motion (see Karatzas and Shreve [21]). Furthermore,

$$
\begin{gathered}
P\left\{\inf _{s \geq t-2 H}\left(u s+c s^{1-\frac{1}{2 H}}+B(s)\right)<0\right\} \leq P\left\{\inf _{s \geq t^{-2 H}}\left(u s+c t^{1-2 H}+B(s)\right)<0\right\} \\
=P\left\{\inf _{s \geq t^{-2 H}}\left(c t^{1-2 H}+u t^{-2 H}+B\left(t^{-2 H}\right)\right)\right. \\
\left.\left.+B(s)-B\left(t^{-2 H}\right)+u\left(s-t^{-2 H}\right)\right)<0\right\}
\end{gathered}
$$

where the first inequality holds because $1 / 2<H<1$. Using Lemma 2 , the last ex- 
pression is equal to

$$
\begin{aligned}
& \frac{t^{H}}{\sqrt{2 \pi}} \int_{-\infty}^{-c t^{1-2 H}-u t-2 H} e^{-\frac{t^{2 H} x^{2}}{2}} d x \\
& +\frac{t^{H}}{\sqrt{2 \pi}} \int_{-c t^{1-2 H}-u t-2 H}^{\infty} e^{-2 u\left(c t^{1-2 H}+u t^{-2 H}+x\right)-\frac{t^{2 H} x^{2}}{2}} d x \\
& =1-\Phi\left(u t^{-H}+c t^{1-H}\right)+\exp \left(-2 u c t^{1-2 H}\right)\left[1-\Phi\left(u t^{-H}-c t^{1-H}\right)\right],
\end{aligned}
$$

which completes the proof.

An immediate consequence of Theorem 2 is the following result known for the Lévy processes.

Corollary 1: Let $B_{H}$ be a fractional Brownian motion process defined in (27) and $u>0$. Then

$$
P\left\{\sup _{0 \leq s \leq t} B_{H}(s)>u\right\} \leq 2 P\left\{B_{H}(t)>u\right\}
$$

Proof: Take $c=0$ in Theorem 2 .

The following theorem enables us to approximate the ruin probability of the process in (30) for a sufficiently large initial capital. $t>0$,

Theorem 6: Let $Q=(Q(t): t \geq 0)$ be the process defined in (30). Then for every

$$
\lim _{u \rightarrow \infty} \frac{P\{(Q) \leq t\}}{P\left\{\lambda^{H} B_{H}(t)>u+c t\right\}}=1,
$$

where the functional $T$ is given in (17).

Proof: For simplicity, assume $\lambda=1$ and $E\left[B_{H}^{2}(1)\right]=1$.

The numerator in (39) is clearly greater than or equal to the denominator. Thus it suffices to show that

$$
\limsup _{u \rightarrow \infty} \frac{P\{T(Q) \leq t\}}{P\left\{B_{H}(t)>u+c t\right\}} \leq 1 .
$$

Let us notice that for $r>0$,

$$
\begin{aligned}
& P\{T(Q) \leq t\} \\
& \leq P\left\{\sup _{0 \leq s<t-\frac{r}{u^{2}}}\left(B_{H}(s)-c s\right)>u\right\}+P\left\{\begin{array}{l}
\sup _{t-\frac{r}{u^{2}} \leq s \leq t}\left(B_{H}(s)-c s\right)>u \\
0
\end{array}\right\} \\
& \leq P\left\{\sup _{0 \leq s \leq t-\frac{r}{u^{2}}}\left(B_{H}(s)-c s\right)>u\right\} \\
& +P\left\{\sup _{t-\frac{r}{u^{2}} \leq s \leq t} B_{H}(s)>u+c\left(t-r / u^{2}\right)\right\} \text {. }
\end{aligned}
$$

First we show that: 


$$
\limsup _{u \rightarrow \infty} \frac{P\left\{\sup _{0} \leq s \leq t-\frac{r}{u^{2}}\left(B_{H}(s)-c s\right)>u\right\}}{P\left\{B_{H}(t)>u+c t\right\}} \leq 2 \exp \left(-r H t^{-2 H-1}\right) .
$$

Using Theorem 2, we may write:

$$
\begin{gathered}
P\left\{\sup _{\left.0 \leq s \leq t-\frac{r}{u^{2}}\left(B_{H}(s)-c s\right)>u\right\}}\right. \\
\leq 1-\Phi\left(\frac{u+c\left(t-\frac{r}{u^{2}}\right)}{\left(t-\frac{r}{u^{2}}\right)^{H}}\right)+\exp \left(\frac{-2 u c\left(t-\frac{r}{u^{2}}\right)}{\left(t-\frac{r}{u^{2}}\right)^{2 H}}\right)\left[1-\Phi\left(\frac{u-c\left(t-\frac{r}{u^{2}}\right)}{\left(t-\frac{r}{u^{2}}\right)^{H}}\right)\right] .
\end{gathered}
$$

Write:

$$
\frac{u+c\left(t-\frac{r}{u^{2}}\right)}{\left(t-\frac{r}{u^{2}}\right)^{H}}=\frac{\left(u+c\left(t-\frac{r}{u^{2}}\right)\right) / t^{H}}{1+\frac{u^{2}\left[\left(t-\frac{r}{u^{2}}\right)^{H}-t^{H}\right]}{u^{2} t^{H}}}
$$

and note that $(1+y)^{-1}=1-y+O\left(y^{2}\right)$ for $y \rightarrow 0$. Then the right-hand member of (43) is equal to:

$$
\frac{u+c\left(t-\frac{r}{u^{2}}\right)}{t^{H}}\left\{1-\frac{u^{2}\left[\left(t-\frac{r}{u^{2}}\right)^{H}-t^{H}\right]}{u^{2} t^{H}}+O\left(\left[\frac{u^{2}\left[\left(t-\frac{r}{u^{2}}\right)^{H}-t^{H}\right]}{u^{2} t^{H}}\right]^{2}\right)\right\}
$$

which, by the existence of the derivative of $t^{H}$, is equal to

$$
\frac{u+c\left(t-\frac{r}{u^{2}}\right)}{t^{H}}+\frac{r H\left(u+c\left(t-\frac{r}{u^{2}}\right)\right)}{u^{2} t^{H+1}}+o\left(\frac{1}{u}\right) .
$$

As a consequence of the estimate in (43) of (42), and (29), it follows that

$$
\begin{aligned}
1-\Phi\left[\frac{u+c\left(t-\frac{r}{u^{2}}\right)}{\left(t-\frac{r}{u^{2}}\right)^{H}}\right] \\
\\
\sim 1-\left[\Phi\left(\frac{u+c\left(t-\frac{r}{u^{2}}\right)}{t^{H}}\right)\right] \exp \left(-r H t^{\left.-2 H-1 \frac{\left[u+c\left(t-\frac{r}{u^{2}}\right)\right]^{2}}{u^{2}}\right)}\right.
\end{aligned}
$$

as $u \rightarrow \infty$. Let us notice that (using (28)),

$$
\exp \left(\frac{-2 u c\left(t-\frac{r}{u^{2}}\right)}{\left(t-\frac{r}{u^{2}}\right)^{2 H}}\right)\left[1-\Phi\left(\frac{u-c\left(t-\frac{r}{u^{2}}\right)}{\left(t-\frac{r}{u^{2}}\right)^{H}}\right)\right]
$$

is asymptotically equal to 


$$
1-\Phi\left(\frac{u+c\left(t-\frac{r}{u^{2}}\right)}{\left(t-\frac{r}{u^{2}}\right)^{H}}\right)
$$

as $u \rightarrow \infty$. So by (42) and (45) we obtain (41).

Now we prove that

$$
\limsup _{u \rightarrow \infty} \frac{P\left\{\sup _{t-\frac{r}{u^{2}} \leq s \leq t} B_{H}(s)>u+c\left(t-r / u^{2}\right)\right\}}{P\left\{B_{H}(t)>u+c t\right\}} \leq 1 .
$$

For the purpose of establishing (46), note that the event in the numerator in (46) is included in the union of the following three events:

and

$$
\begin{gathered}
B_{H}(t)>u+c\left(t-\frac{r}{u^{2}}\right) \\
\sup _{t-\frac{r}{u^{2}} \leq s \leq t} B_{H}(s)>u+c\left(t-\frac{r}{u^{2}}\right), \quad B_{H}(t) \leq 0 ;
\end{gathered}
$$

$$
\sup _{t-\frac{r}{u^{2}} \leq s \leq t} B_{H}(s)>u+c\left(t-\frac{r}{u^{2}}\right), \quad 0<B_{H}(t) u+c\left(t-\frac{r}{u^{2}}\right) ;
$$

Using the method given in Berman [6], verification of (2.6) (see also Berman [5]), we get that the probability of the events in (48) and (49) are of smaller order than $1-\Phi\left(\frac{u+c t}{t^{H}}\right)$. The probability of (47) is asymptotically equal to $P\left\{B_{H}(T)>u+c t\right\}$ as $u \rightarrow \infty$, which completes the proof of the theorem.

So for sufficiently large $u$, we may write:

$$
P\left\{\inf _{0 \leq s \leq t}\left(u+c s-\lambda^{H} B_{H}(s)\right)<0\right\} \approx P\left\{\lambda^{H} B_{H}(t)>u+c t\right\} .
$$

Let us notice that in the case when we approximate risk process by Brownian motion with drift (see Iglehart [20]), then

$$
\lim _{u \rightarrow \infty} \frac{P\left\{\inf _{0} \leq s \leq t\right.}{P\left\{\lambda^{1 / 2} B(t)>u+c t\right\}}=2,
$$

where $B$ is a Brownian motion. In the model with dependent claims, the situation is changing drastically, and the ruin probability is greater than in the Brownian case, that is,

$$
P\left\{\lambda^{H} B_{H}(t)>u+c t\right\}>2 P\left\{\lambda^{1 / 2} B(t)>u+c t\right\}
$$

for all $t>t_{0}$ where $t_{0}>0$. 


\section{Numerical Results}

In order to get some appreciation of the behavior of the process $Q$ defined in (30), we compute the ruin probability for fractional Brownian motion using the simple Monte Carlo method and compare it with the upper bound given in (33). The lower bound, which should be closer to the real ruin probability, is also considered. Fractional Brownian motion was simulated with the well-known and reliable method of Cholesky factorization (see Rice [34]). This method can be used for estimation of continuous functionals of a sample (e.g., extremes) with a predetermined error. One should write that for continuous but nondifferentiable Gaussian processes, the method needs a fine grid (leading to long execution times) for reliable results (see Seleznjev [40]). But is was shown in Seleznjev [39], that for general classes of random processes with incremental variance,

$$
E\left[(X(t)-X(s))^{2}\right] \leq C|t-s|^{\alpha}, \quad C>0, \alpha>0,
$$

with the best rate of approximation in the quadratic mean being $n^{-\alpha / 2}$, where $n$ is a number of used linear functionals (e.g., values of the process $X\left(t_{k}\right), k=1, \ldots$, ).

Let

$$
\Psi(u, t)=P\left\{\inf _{0 \leq s \leq t}\left(u+c s-\lambda^{H} B_{H}(s)\right)<0\right\},
$$

assume $\sigma^{2}=E\left[B_{H}^{2}(1)\right]$ and

$$
\Psi_{U}(u, t)=1-\Phi\left(\frac{u+c t}{\sigma(\lambda t)^{H}}\right)+\exp \left(\frac{-2 u c t}{\sigma^{2}(\lambda t)^{2 H}}\right)\left[1-\Phi\left(\frac{u-c t}{\sigma(\lambda t)^{H}}\right)\right]
$$

which is the upper bound to $\Psi(u, t)$. The lower bound is

$$
\Psi_{L}(u, t)=1-\Phi\left(\frac{u+c t}{\sigma(\lambda t)^{H}}\right)
$$

In Table 1, we have presented some numerical values for illustrative purposes. In our simulations, $H \in 0.6,0.8,0.9$, the latter values are $t=5, c=1, \lambda=1, \sigma=10$. we divided the interval $[0, t]$ according to 1500 points (e.g., we took 1500 random variables to compute the ruin probability). We ran 40000 simulations in order to estimate $\Psi(u, t)$. The half-width of the asymptotic $95 \%$ confidence interval is denoted by $\epsilon$.

As has been shown in Theorem 6, it follows from our simulations that the ruin probability for fractional Brownian motion is very close to the lower bound. The upper bound also gives a good approximation. For large $u$, the upper bound behaves like $2\left(1-\Phi\left(\frac{u+c t}{\sigma(\lambda t)^{H}}\right)\right)$, which the numerical results show.

The ruin probability is strongly dependent on the parameter $H$ of self-similarity, and the ruin probability is larger when the dependence of increments (or claims) is stronger. 


\begin{tabular}{|c|c|c|c|c|c|}
\hline$u$ & $H$ & $\Psi(u, t)$ & $\Psi_{L}(u, t)$ & $\Psi_{U}(u, t)$ & $\epsilon$ \\
\hline 30 & 0.6 & 0.1456 & 0.0913 & 0.2018 & 0.0035 \\
\hline 60 & 0.6 & 0.0098 & 0.0067 & 0.0143 & $9.6662 \cdot 10^{-4}$ \\
\hline 100 & 0.6 & $5.0000 \cdot 10^{-5}$ & $3.1984 \cdot 10^{-5}$ & $6.6963 \cdot 10^{-5}$ & $6.9296 \cdot 10^{-5}$ \\
\hline 30 & 0.8 & 0.1938 & 0.1671 & 0.3621 & 0.0039 \\
\hline 60 & 0.8 & 0.0382 & 0.0364 & 0.0773 & 0.0019 \\
\hline 100 & 0.8 & 0.0019 & 0.0019 & 0.0039 & $4.1828 \cdot 10^{-4}$ \\
\hline 30 & 0.9 & 0.2168 & 0.2055 & 0.415 & 0.0040 \\
\hline 60 & 0.9 & 0.0639 & 0.0634 & 0.1339 & 0.0024 \\
\hline 100 & 0.9 & 0.0069 & 0.0068 & 0.0142 & $8.1125 \cdot 10^{-4}$ \\
\hline
\end{tabular}

Table 1: Comparison of the finite-time ruin probabilities of the fractional Brownian motion $(\lambda=1, c=1, \sigma=10)$ with the upper and lower bound.

The time horizon $t$ equals 5 .

\section{Acknowledgments}

The author would like to thank Igor Rychlik for many useful and stimulating discussions related to the extreme value theory of Gaussian processes. The author also takes pleasure in thanking Søren Asmussen for his many constructive comments and suggestions.

\section{References}

[1] Asmussen, S., Approximations for the probability of ruin within finite time, Scan. Actuarial J. (1984), 31-57.

[2] Asmussen, S., Applied Probability and Queues, John Wiley and Sons, New York 1987.

[3] Asmussen, S., Ruin Probabilities, manuscript 1995.

[4] Asmussen, S. and Michna, Z., Simulation of ruin probabilities for fractional Brownian motion, working paper (1997).

[5] Berman, S.M., Sojourns and extremes of stationary processes, Ann. Prob. 10 (1982), 1-46.

[6] Berman, S.M., An asymptotic formula for the distribution of the maximum of a Gaussian process with stationary increments, J. Appl. Prob. (1985), 454-460.

[7] Billingsley, P., Convergence to Probability Measures, John Wiley and Sons, New York 1968.

[8] Cramer, H., Collective risk theory, Jubilee Vol. of Forsakringsaktiebolaget Skandia, Nordiska Bokhandeln, Stockholm (1955), 1-92.

[9] Crovella, M. and Bestavros, A., Explaining world wide web traffic self-similarity, Preprint available as TR-95-015 from crovella.best@cs.bu.edu (1995). 
[10] Cunha, M., Bestavros, A. and Crovella, M., Characteristics of www client-based traces, Preprint available as BU-CS-95-101 from crovella.best@cs.bu.edu (1995).

[11] Embrechts, P. and Schmidli, H., Modelling of extremal events in insurance and finance, ZOR - Math. Methods Oper. Res. 39 (1994), 1-34.

[12] Embrechts, P. and Schmidli, H., Ruin estimation for a general insurance risk model, Adv. in Appl. Probab. 26 (1994), 404-422.

[13] Erramilli, A., Narayan, O. and Willinger, W., Experimental queueing analysis with long-range dependent packet traffic, Preprint, Bellcore, Morristown, NY 07960 (1995).

[14] Ethier, S.N. and Kurtz, T.G., Markov Processes, John Wiley and Sons, New York 1986.

[15] Feller, W., An Introduction to Probability Theory and its Applications, Vol. II, John Wiley and Sons, New York 1971.

[16] Furrer, H., Michna, Z.and Weron, A., Stable Lévy motion approximation in collective risk theory, Insurance: Mathematics and Economics 20 (1997), 97-114.

[17] Grandell, J., Aspects of Risk Theory, Springer-Verlag, New York 1991.

[18] Heath, D., Resnick, S. and Samorodnitsky, G., Heavy tails and long range dependence in on/off processes and associated fluid models, Preprint (1996).

[19] Hadwiger, H., Über die Wahrscheinlichkeit des Ruins bei einer grossen Zahl von Geschaften, Archiv v. mathematische Wirstchaft - und Sozialforschung 6 (1940), 131-135.

[20] Iglehart, D.L., Diffusion approximation in collective risk theory, J. Appl. Prob. 6 (1969), 285-292.

[21] Karatzas, I. and Shreve, S.E., Brownian Motion and Stochastic Calculus, Second Ed., Springer-Verlag, New York 1988.

[22] Lamperti, J., Semi-stable stochastic processes, Trans. Amer. Math. Soc. 104 (1962), 62-78.

[23] Leadbetter, M.R., Lindgren, G. and Rootzén, H., Extremes and Related Properties of Random Sequences and Processes, Springer-Verlag, New York 1983.

[24] Leland, W., Lan traffic behavior from milliseconds to days, In: 7th ITC Specialists' Seminar, Morristown (1990).

[25] Leland, W. and Wilson, D., High time-resolution measurement and analysis of LAN traffic: Implications for LAN interconnection, In: IEEE INFOCOM'91 (1991).

[26] Leland, W., Taqqu, M., Wallinger, W. and Wilson, D., On the self-similar nature of ethernet traffic, Proc. of the ACM/SIGCOMM '93, ACM/SIGCOMM Comp. Commun. Rev. 23 (1993), 183-193.

[27] Leland, W., Taqqu, M., Willinger, W. and Wilson, D., On the self-similar nature of ethernet traffic (extended version), IEEE/ACM Trans. on Networking 2 (1994), 1-15.

[28] Livney, M., Melamed, B. and Tsiolis, A.K., The impact of autocorrelations on queueing systems, Management Sci. 39 (1993), 322-339.

[29] Mandelbrot, B.B. and Van Ness, J., Fractional Brownian motions, fractional noises and applications, SIAM Rev. 10 (1968), 422-427.

[30] Norros, I., A storage model with self-similar input, Queueing Systems 16 (1994), 387-396.

[31] Norros, I., On the use of fractional Brownian motion in the theory of connectionless networks, IEEE J. on Selected Areas in Commun. 13:6 (1995). 
[32] Norros, I., Four Approaches to the Fractional Brownian Storage, Fractals in Eng., Springer 1997.

[33] Resnick, S. and Samorodnitsky, G., Performance decay in a single server exponential queueing model with long range dependence, Oper. Res. (1997), 235-243.

[34] Rice, J., Matrix Computations and Mathematical Software, McGraw-Hill, Tokyo 1983.

[35] Rosenblatt, M., Independence and dependence, In: Proc. Fourth Berkeley Symp. on Math. Stats. and Prob. II, University of California Press, Berkeley (1961), 431-443.

[36] Samorodnitsky, G. and Taqqu, M.S., Stable Non-Gaussian Random Processes, Chapmen and Hall, London 1994.

[37] Schmidli, H.P., A general insurance risk model, Ph.D. Thesis, ETH Zurich 1992.

[38] Schmidli, H.P., Diffusion approximations for a risk process with the possibility of borrowing and investment, Commun. in Stats. - Stoch. Models 10:2 (1994), 365-388.

[39] Seleznjev, O.V., The best approximation of random processes and approximation of periodic random processes, Univ. Lund. Stat. Res. Rep. 6 (1989), 1-32.

[40] Seleznjev, O.V., Limit theorems for maxima of a sequence of Gaussian nonstationary processes and interpolation of random processes, Univ. Lund Stat. Res. Rep. 7 (1993), 1-14.

[41] Seleznjev, O.V., Large deviations in the piecewise linear approximation of Gaussian processes with stationary increments, Adv. Appl. Prob. 28 (1996), 481499.

[42] Slepian, D., The one-sided barrier problem for Gaussian noise, Bell Sys. Tech. J. 41 (1962), 463-501.

[43] Stone, C., Weak convergence of stochastic processes defined on semi-infinite time intervals, Proc. Amer. Math. Soc. 14 (1963), 694-696.

[44] Takács, L., Combinatorial Methods in the Theory of Stochastic Processes, John Wiley and Sons, New York 1967.

[45] Taqqu, M.S., Limit theorems for sums of strongly dependent random variables, Ph.D. Thesis, Columbia Univ. 1972.

[46] Taqqu, M.S. Weak convergence to fractional Brownian motion and to the Rosenblatt process, Z. Wahrsch. verw. Gebiete 31 (1975), 287-302.

[47] Willinger, W., Taqqu, M., Leland, W. and Wilson, D., Self-similarity in highspeed packet traffic: analysis and modeling of ethernet traffic measurements, Stat. Sci. 10 (1995), 67-85.

[48] Willinger, W., Taqqu, M., Sherman, R. and Wilson, D., Self-similarity through high-variability: statistical analysis of ethernet LAN traffic at the source level, Preprint (1995). 


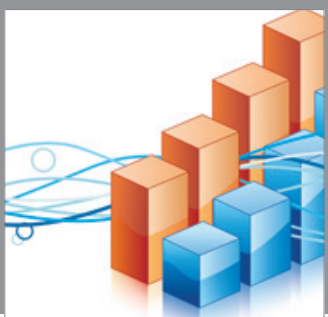

Advances in

Operations Research

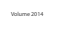

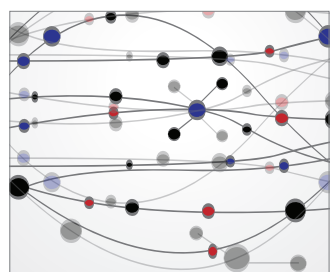

\section{The Scientific} World Journal
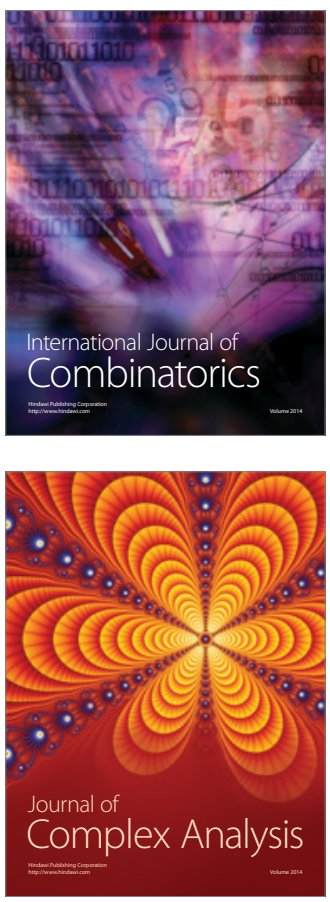

International Journal of

Mathematics and

Mathematical

Sciences
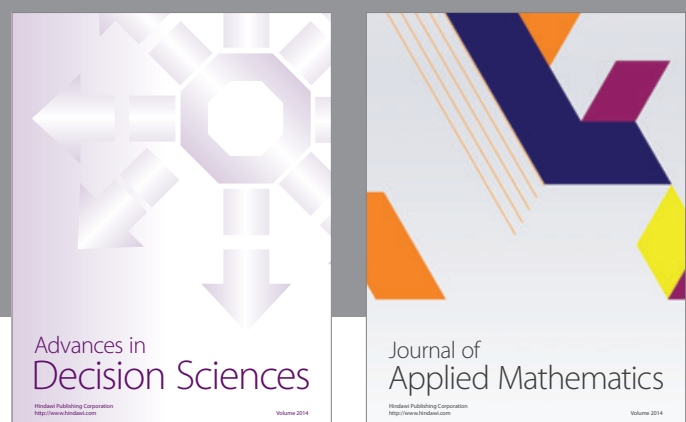

Journal of

Applied Mathematics
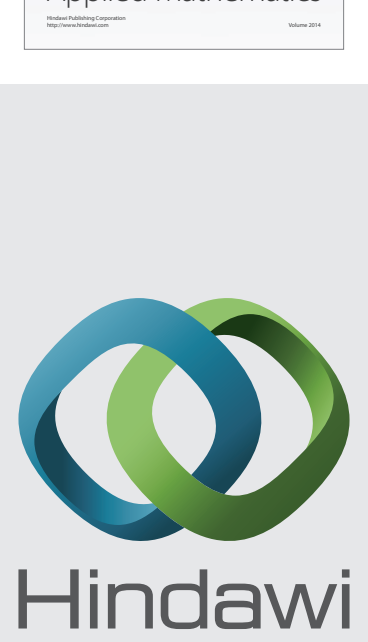

Submit your manuscripts at http://www.hindawi.com
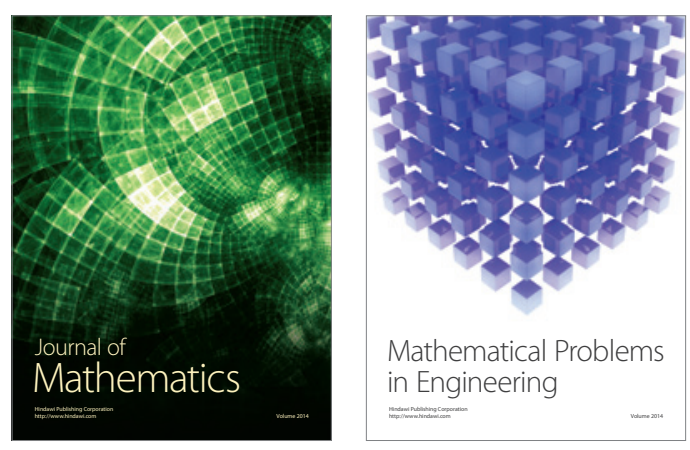

Mathematical Problems in Engineering
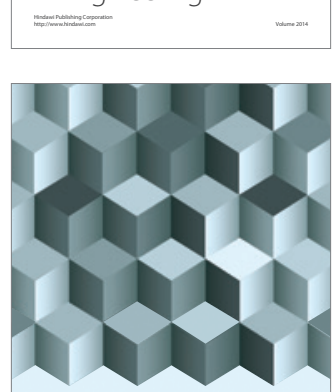

Journal of

Function Spaces
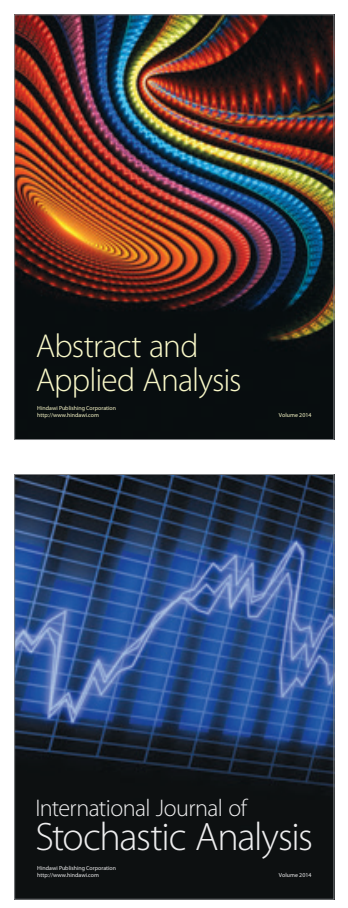

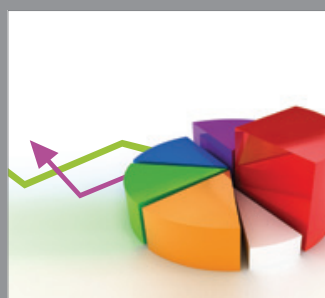

ournal of

Probability and Statistics

Promensencen
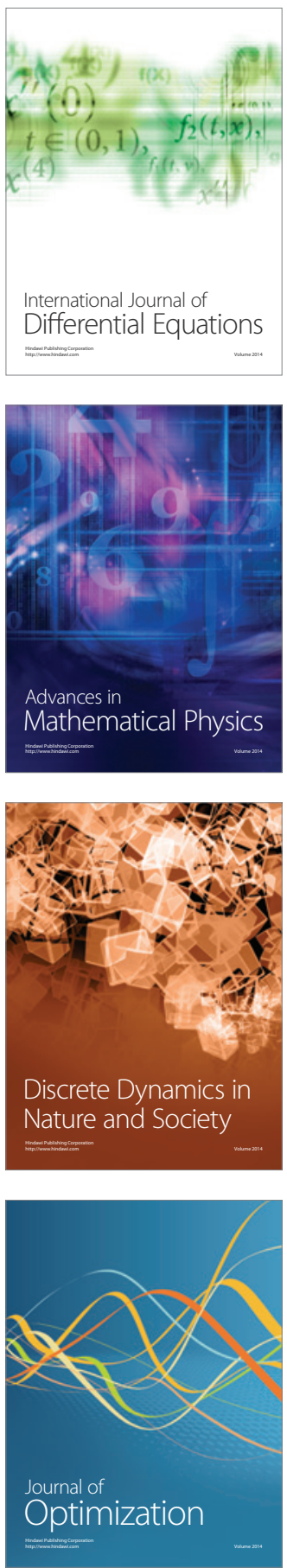